\title{
Quantized charge transport driven by a surface acoustic wave in induced unipolar and bipolar junctions
}

\author{
Yousun Chung $\odot,{ }^{1, *}$ Hangtian Hou, ${ }^{1}$ Seok-Kyun Son $\odot,{ }^{1, \dagger}$ Tzu-Kan Hsiao, ${ }^{1, \ddagger}$ Ateeq Nasir $\odot,{ }^{1,2}$ Antonio Rubino, ${ }^{1}$ \\ Jonathan P. Griffiths, ${ }^{1}$ Ian Farrer $\odot,{ }^{1,}{ }^{\S}$ David A. Ritchie, ${ }^{1}$ and Christopher J. B. Ford ${ }^{1, \|}$ \\ ${ }^{1}$ Cavendish Laboratory, University of Cambridge, Cambridge CB3 OHE, United Kingdom \\ ${ }^{2}$ National Physical Laboratory, Hampton Road, Teddington TW11 OLW, United Kingdom
}

(Received 4 June 2019; revised manuscript received 27 October 2019; published 2 December 2019)

\begin{abstract}
Surface acoustic waves (SAWs) have been used to transport single electrons across long distances of several hundreds of microns. They can potentially be instrumental in the implementation of scalable quantum processors and quantum repeaters, by facilitating interaction between distant qubits. While most of the work thus far has focused on SAW devices in doped GaAs/AlGaAs heterostructures, we have developed a method of creating lateral $p-n$ junctions in an undoped heterostructure containing a quantum well, with the expected advantages of having reduced charge noise and increased spin-coherence lifetimes due to the lack of dopant scattering centers. We present experimental observations of SAW-driven single-electron quantized current in an undoped $\mathrm{GaAs} / \mathrm{AlGaAs}$ heterostructure, where single electrons were transported between regions of induced electrons. We also demonstrate pumping of electrons by a SAW across the submicron depleted channel between regions of electrons and holes, and observe light emission at such a lateral $p-n$ junction. Improving the lateral confinement in the junction should make it possible to produce a quantized electron-to-hole current and hence SAW-driven emission of single photons.
\end{abstract}

DOI: 10.1103/PhysRevB.100.245401

\section{INTRODUCTION}

For quantum cryptography and quantum information processing, a solid-state single-photon source capable of providing a controllable number of photons on demand with a high repetition rate is required [1]. Foden et al. [2] proposed that the stream of single electrons that can be driven by a surface acoustic wave (SAW) through a semiconductor channel could be directed into a sea of holes and hence generate single photons. The number of photons emitted should equal the number of electrons trapped in each SAW minimum, while the polarization of the emitted photons depends on the spin state of the incoming carriers. It should therefore be possible to use SAWs to facilitate long-range interactions between qubits not only by physically transporting qubits in the SAW minima [3-11], but also by transferring spin information via SAW-generated polarized single photons [12].

\footnotetext{
*yousun.chung@unsw.edu.au; Present address: Centre of Excellence for Quantum Computation and Communication Technology, University of New South Wales, Sydney, New South Wales 2052, Australia.

${ }^{\dagger}$ Present address: Department of Physics, Mokpo National University, Muan 58554, Republic of Korea.

${ }^{\ddagger}$ Current Address: QuTech, Delft University of Technology, P.O. Box 5046, 2600 GA Delft, The Netherlands.

§Present address: Department of Electronic and Electrical Engineering, The University of Sheffield, Sheffield S1 3JD, United Kingdom.

"cjbf@cam.ac.uk
}

We have previously shown that a SAW could carry a welldefined small number of electrons through a short pinched-off channel in a modulation-doped GaAs/AlGaAs heterostructure [13,14], by observing plateaus in the SAW-driven current as a function of the voltage on a split pair of gates on either side of the channel. The plateaus, of height $I=N e f$ (where $e$ is electronic charge and $f$ is the SAW frequency), correspond to transport through the channel of an integer number $N$ of electrons trapped in each SAW minimum. $N$ is determined by the SAW potential and the maximum electrostatic potential gradient in the one-dimensional (1D) channel.

To emit single photons, such a SAW-driven stream of electrons must be dragged into a $p$-type region for recombination with holes. However, fabricating lateral devices with both $n$ - and $p$-type regions is nontrivial [15-22]. Electroluminescence (EL) has previously been observed from some lateral $p-n$ junctions $[16,20,22]$. SAW-driven light emission from a lateral $p-n$ junction has also been achieved, but this either required very complicated fabrication processes when using doped substrates (regrowth on patterned substrates) and wide channels [21], or else electrons and holes were induced in undoped material by passing high currents within the separate regions, and there was no sign of quantization of the $n$-to- $p$ current [23].

In this article we present the observation of SAW-induced quantized electron transport in an undoped system, where the use of realistic boundary conditions in self-consistent electrostatic potential calculations was vital in designing the device gate structure. We also demonstrate SAW-driven light emission from a $p-n$ junction formed by inducing controllable equilibrium populations of electrons and holes in close 
proximity in an undoped heterostructure using the same device design. We have thus developed the necessary ingredients for, and also demonstrated the first steps towards, a SAWdriven single-photon source.

\section{EXPERIMENTAL DETAILS}

Our device consists of lateral $p$ - $n$ junctions fabricated on MBE-grown undoped GaAs/AlGaAs heterostructures with a quantum well of width $35 \mathrm{~nm}$ (device A) or $15 \mathrm{~nm}$ (device B) located $110 \mathrm{~nm}$ below the surface. Figure 1 shows the device layout, where depending on the applied voltages, electrons or holes can be induced, allowing permutations of lateral $n-i-n, p-i-p$, or $n-i-p$ junctions in the active region [Fig. 1(b)]. A single junction includes source and drain contacts [see Fig. 1(c)], and two side gates to control the confinement of carriers in the 1D channel. As seen in Figs. 1(d) and 1(e), the bridging gates $(\mathrm{BG})$ overlap with the ohmic contacts, and are used to draw electrons or holes out of the contacts into the well by applying a high bias. The surface gates (SG), which extend a little under the bridging gates, take over the task of inducing carriers, and provide the important ability to control the carrier density at the center of the chip independently of inducing
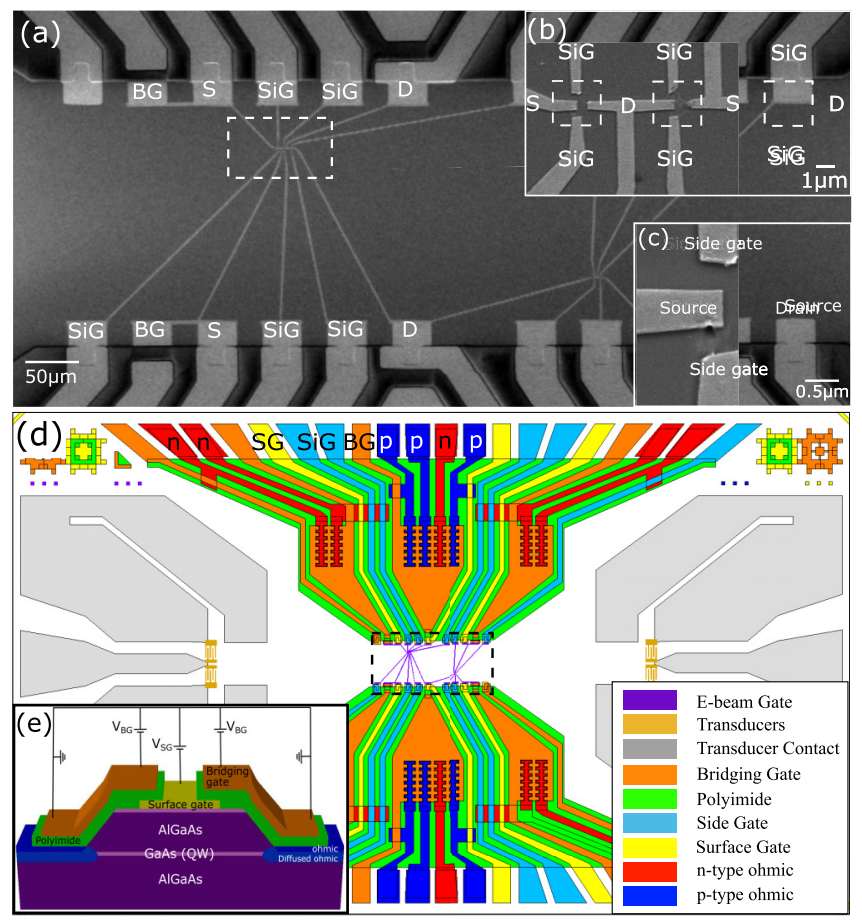

FIG. 1. (a) Scanning-electron microscope (SEM) image of the active region of device $\mathrm{A} . \mathrm{S}, \mathrm{D}, \mathrm{BG}$, and $\mathrm{SiG}$ are source and drain surface gates, bridging gates, and side gates, respectively. Insulated BGs overlap the ohmic contacts, inducing carriers and leading them to the surface inducing gates. D can be used as an $n$ - or $p$-type contact depending on the voltages applied to the corresponding bridging and surface gates. (b) SEM image of the region with three junctions. (c) One of the lateral $n-i-n, p-i-p$, or $n-i-p$ junctions. (d) Schematic of the whole device, where the corresponding contacts have been labeled. More details regarding device operation can be found in the Appendix. (e) Cross section of device, showing the quantum well and the gate stack. charge at the ohmic contacts. Electrons or holes induced under the surface gates extend into the path of the SAW, though the gate size is kept to a minimum to reduce screening of the SAW potential. We aim to have the length of the intrinsic region between source and drain $(\sim 600 \mathrm{~nm})$ greater than half the SAW wavelength so that each SAW potential minimum becomes completely isolated from both leads at some point in the cycle. Using the results of our self-consistent electrostatic modeling, we chose the geometry and dimensions so that SAW transport across the junction in our devices is likely to be quantized by having a strongly confined SAW minimum. In order to generate SAWs, a high-frequency signal is applied to an interdigital transducer (IDT). The IDT periodicity of $1 \mu \mathrm{m}$ gives a SAW resonant frequency of $2.848 \mathrm{GHz}$.

The $p$-type ohmic contacts were defined by optical lithography and the GaAs recessed by wet-etching down close to the quantum well before thermal evaporation of $\mathrm{AuBe}(180 \mathrm{~nm})$, lift-off, and annealing at $520^{\circ} \mathrm{C}$ for $90 \mathrm{~s}$ in order for the dopants to diffuse into the quantum well. Similarly, the $n$-type metallization was achieved by depositing $\mathrm{Ni} / \mathrm{Au} / \mathrm{Ge} / \mathrm{Ni} / \mathrm{Au}$ $(5 / 132 / 66 / 50 / 40 \mathrm{~nm})$ in a self-aligned recess (down to the quantum well), and thermally annealing at a temperature of $460{ }^{\circ} \mathrm{C}$ for 80 s. Polyimide insulator (HD4014) was spin coated and hard baked in a curing oven, providing a thickness of about 300-700 nm. There are three types of gates as shown in Fig. 1(d)-bridging, surface, and side gates. The bridging gates were patterned optically and thermally evaporated with $\mathrm{Ti} / \mathrm{Au}(20 / 80 \mathrm{~nm})$, while $\mathrm{Ti} / \mathrm{Au}(7 / 10 \mathrm{~nm})$ surface and side gates were patterned using electron-beam lithography.

\section{RESULTS AND DISCUSSIONS}

For the $n-i-n$ junction devices discussed in this paper, the direction of the SAW is from drain to source, so that electrons are transported in this direction across the intrinsic region when the SAW amplitude is large enough that each SAW potential minimum retains a dip when added to the steep potential in the intrinsic region. Figure 2 shows the SAW-driven electron current across an $n-i-n$ junction. Below $11 \mathrm{dBm}$ there was no current at the resonant frequency [see Fig. 2(a)], implying that a SAW power of $11 \mathrm{dBm}$ was not enough to overcome the slope of the potential barrier in the intrinsic region and hence transport electrons across the junction. Above $11 \mathrm{dBm}$, the current increased strongly with power. The source and drain currents were equal and opposite, showing that there was no leakage current. Note that for this measurement, there was no applied bias on the side gates, and also no external S-D bias, so the current was SAW-driven transport directly across the intrinsic region. This is further supported by bias-dependence measurements, which show that the current is independent of S-D bias in the range $\pm 5 \mathrm{mV}$. This is consistent with SAW-driven transport without significant contributions from crosstalk or any conduction path through the side gates due to an external source-drain bias or side-gate voltage.

Figure 2(c) shows experimental evidence of SAW quantization for voltages on the surface gates above the threshold at which carriers are induced under them. The measured current vs side-gate bias shows quantized plateaus, as $N$ decreases, and eventually the SAW current goes to zero when the 

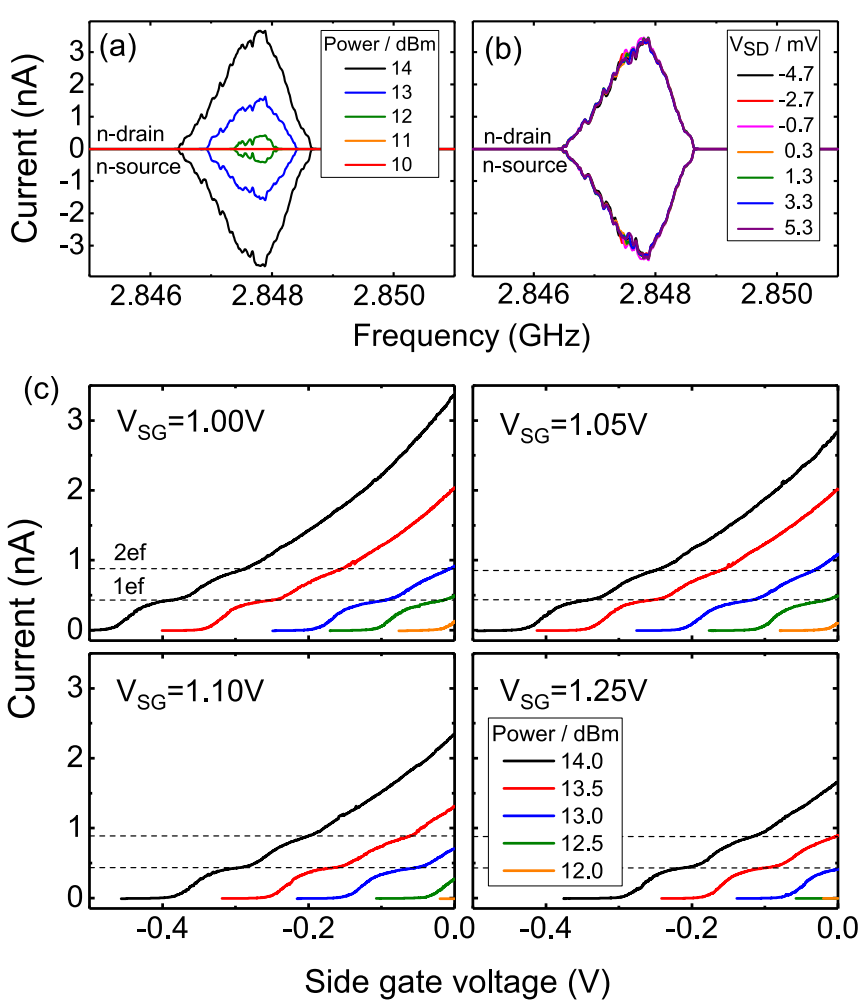

FIG. 2. The acoustoelectric current $I_{\mathrm{ae}}$ of electrons driven by a SAW across an $n-i-n$ junction from $\mathrm{S}$ to $\mathrm{D}$ vs SAW frequency $f$ (device A). D and $\mathrm{S}$ currents are equal and opposite. $V_{\mathrm{SG}}=1.1 \mathrm{~V}$, $V_{\mathrm{SiG}}=0 \mathrm{~V}, T=4.2 \mathrm{~K}$. (a) $I_{\mathrm{ae}}$ varying $\mathrm{SAW}$ power from 14 to $10 \mathrm{dBm}$. (b) $I_{\mathrm{ae}}$ varying $\mathrm{S}-\mathrm{D}$ bias $V_{\mathrm{SD}}$ at $14 \mathrm{dBm}$ SAW power, $V_{\mathrm{BG}}=2.2 \mathrm{~V}, V_{\mathrm{SG}}=1.15 \mathrm{~V}, V_{\mathrm{SiG}}=0 \mathrm{~V}$. (c) $I_{\mathrm{ae}}$ across the $n-i-n$ junction vs side-gate voltage $V_{\mathrm{SiG}}$ at different SAW powers and surface-gate voltages $V_{\mathrm{SG}}$, showing approximately quantized current plateaus close to multiples of $e f=456 \mathrm{pA}(f=2.848 \mathrm{GHz})$.

potential is too steep for the SAW to pump any electrons. The SAW power and surface gate (and hence the carrier density) were varied in order to try to optimize the confinement of the moving quantum dot, and hence increase the chance of observing quantization, though little improvement was found. Two plateaus are visible, where the first plateau is found at $0.45 \mathrm{nA}$ and the second at $0.9 \mathrm{nA}$, in agreement with expectations for a SAW-pumped single-electron current following the equation $I=N e f$. Probably partly owing to the measurement temperature used $(T=4.2 \mathrm{~K})$, the plateaus are not as flat as can be achieved in doped devices [24]. Nevertheless, our results show that it is possible to transfer a well-defined number $N$ of electrons in each SAW minimum and to control it using side-gate voltages. This is a significant step towards creating a single-photon source using an undoped induced $n-i$ - $p$ junction device, in which a single electron would be transferred by the SAW in each cycle and would recombine with one of the holes induced on the other side of the junction. We present preliminary results on SAW-driven current and electroluminescence in the second part of this paper.

We now discuss the effect of gate geometry on the quantization of the SAW-driven current. In developing an electrostatic model for undoped GaAs devices, it is important to note that, unlike in doped devices, assigning the correct

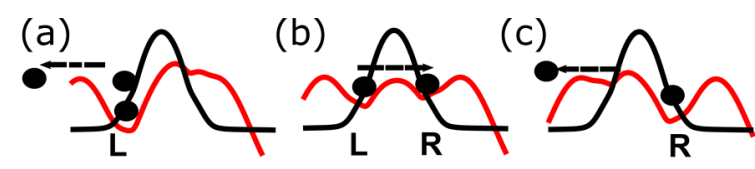

(d)

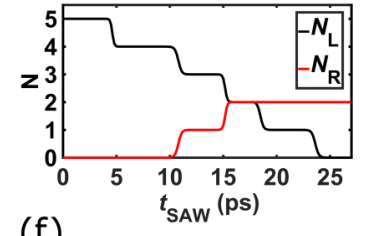

(f)

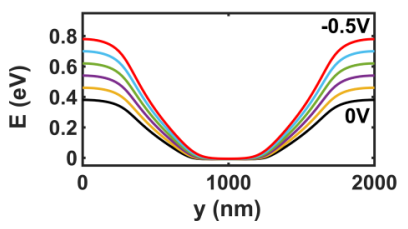

(e)

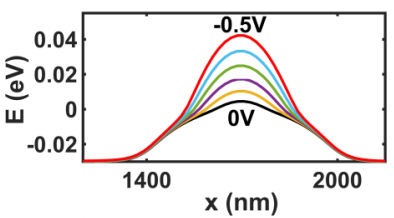

(g)

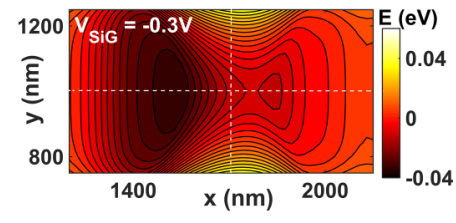

FIG. 3. (a)-(c) Model $n-i-n$ potential energy in the $x$ direction (black lines) and with an added SAW (red lines) at different times in a SAW cycle. Black dots are electrons trapped in potential minima, before and after the tunneling events shown by arrows. (d) The number of electrons $N_{\mathrm{L}}$ and $N_{\mathrm{R}}$ in the two minima seen in (a)-(c) as a function of time $t_{\mathrm{SAW}}$ as the SAW travels through the junction. (e) Cut through the potential energy in the $y$ direction, along the white dashed line shown in $(\mathrm{g})$ for $V_{\mathrm{Sig}}$ from 0 to $-0.5 \mathrm{~V}$ in steps of $0.1 \mathrm{~V}$, as labeled. (f) Cut through the potential energy in the $x$ direction, along the white dashed line shown in $(\mathrm{g})$ for $V_{\mathrm{SiG}}$ from 0 to $-0.5 \mathrm{~V}$ in steps of $0.1 \mathrm{~V}$, as labeled. $(\mathrm{g})$ The electrostatic potential energy in the $x-y$ plane at the depth of the QW in an $n-i-n$ junction at side-gate voltage $V_{\mathrm{SiG}}=-0.3 \mathrm{~V}$, with an added screened $\mathrm{SAW}$ potential of peak amplitude $25 \mathrm{meV}$ at $t_{\mathrm{SAW}}=10 \mathrm{ps}$.

boundary conditions is critical and nontrivial, because of the absence of modulation doping, and hence of built-in electric fields. In a doped GaAs device it is common to assume that the Fermi energy is pinned at the regrowth interface $\sim 1 \mu \mathrm{m}$ below the surface. By comparing the model and experiments, we have shown that this boundary condition is inappropriate as charges in dangling bonds there become frozen at cryogenic temperatures, and a deep boundary condition in the GaAs substrate gives more accurate results, and indeed we have shown that it is essential in calculations for an undoped device [25]. Figure $3(\mathrm{~g})$ shows the calculated electrostatic potential energy in an induced $n-i-n$ junction matching the experimental design. The junction entrance presents a potential slope of $\sim 20-30 \mathrm{meV} / \mu \mathrm{m}$, which the SAW is expected to be able to overcome with applied SAW power above $10-15 \mathrm{dBm}$ [26,27], comparable to our experimental results in Fig. 2. In the experiment, the side-gate pinch-off voltage becomes more negative with increasing SAW power because the higher SAW amplitude enables pumping up the steeper slope. This voltage ranges from 0 to $-0.4 \mathrm{~V}$ for powers ranging from 12 to $14 \mathrm{dBm}$, in agreement with the simulation results. As the side-gate voltage becomes more negative, the transverse confinement becomes stronger and this, together with the SAW longitudinal confinement, defines a dynamic quantum dot. For a given voltage there is some $N$ for which, with $(N+1)$ electrons in the dynamic quantum dot, Coulomb charging of the dot brings the highest-energy electron's energy 
close to the top of the back tunnel barrier and so it is highly likely that the electron tunnels or falls out of the dot back into the original lead, leaving $N$ electrons in the dot to reach the other lead, and this produces a quantized SAW-driven current. We estimate the charging energy to be $\sim 3 \mathrm{meV}$ from our previous work [28].

We use a simple model to calculate the acoustoelectric current through the channel, treating the potential barriers before and after the dot as saddle points with known tunneling-probability functions [29]. Figures 3(a)-3(c) show the calculated potential without (black) and with (red) an applied SAW potential (partially screened in the leads), at various stages of the SAW propagation. First, many electrons collect in the minimum before the barrier [labeled L in Fig. 3(a)], and as the SAW propagates towards the intrinsic barrier, the minimum starts to shrink and some electrons tunnel back into the source [Fig. 3(a)]. As the SAW continues propagating, the intrinsic barrier becomes lower than the following SAW maximum [Fig. 3(b)] so electrons start to tunnel across the barrier and reach the minimum on the right $(\mathrm{R})$ and hence the right lead. Later the intrinsic barrier becomes high again, and electrons remaining in minimum $L$ go back to the source again [Fig. 3(c)], matching the experiment. Figure 3(d) shows the calculated number of electrons $N_{\mathrm{L}}$ and $N_{\mathrm{R}}$ in minima $\mathrm{L}$ and $\mathrm{R}$ seen in Figs. 3(a)-3(c) as a function of time as one wavelength of the SAW travels through the junction. In this case, $N_{\mathrm{R}}=2$ at the end of the cycle, resulting in a quantized acoustoelectric current $I=2 e f$.

We now turn our attention to device operation with different types of carrier induced on each side (forming an $n-i-p$ junction). As a precursor to a SAW-driven singlephoton source, we demonstrate SAW-driven electroluminescence (EL) generated by electrons (holes) from an $n$-type ( $p$-type) region transported by the SAW across the intrinsic region recombining with the induced holes (electrons) in the $p$-type ( $n$-type) region there. As the chemical potentials in the $\mathrm{S}$ and $\mathrm{D}$ leads lie in different bands (valence and conduction), there is a repulsive potential slope in between $\mathrm{S}$ and $\mathrm{D}$. One can forward bias the junction close to the flat-band condition to drive a current and this is likely to give photon emission near the junction even without a SAW, but there is no reason for the photons to come out singly. It should be possible to use SAWs to transport carriers across the intrinsic region with a smaller S-D bias (less than the band gap), just high enough to help the SAW overcome the potential slope between $\mathrm{S}$ and D but not enough to transport charge without a SAW. Then, if the channel is pinched off enough to allow only one electron (hole) per SAW minimum, this single charge will recombine to produce a single photon, ideally before another charge arrives in the next cycle.

EL measurements [see Figs. 4(a) and 4(b)] without a SAW were performed on a second device $(\mathrm{B}$, where some shallow etching was used to confine electrons near to the gates, and using a wafer with a smaller QW width), as a means of confirming that the $p$ - and $n$-type regions were induced at the correct interface. Photon emission was observed under a high $(2.5 \mathrm{~V})$ applied bias. The strongest EL is observed from a 6-10 $\mu$ m-diameter region at the junction, though the resolution of the image is low so the emission spot may actually be smaller than this. The additional EL around the
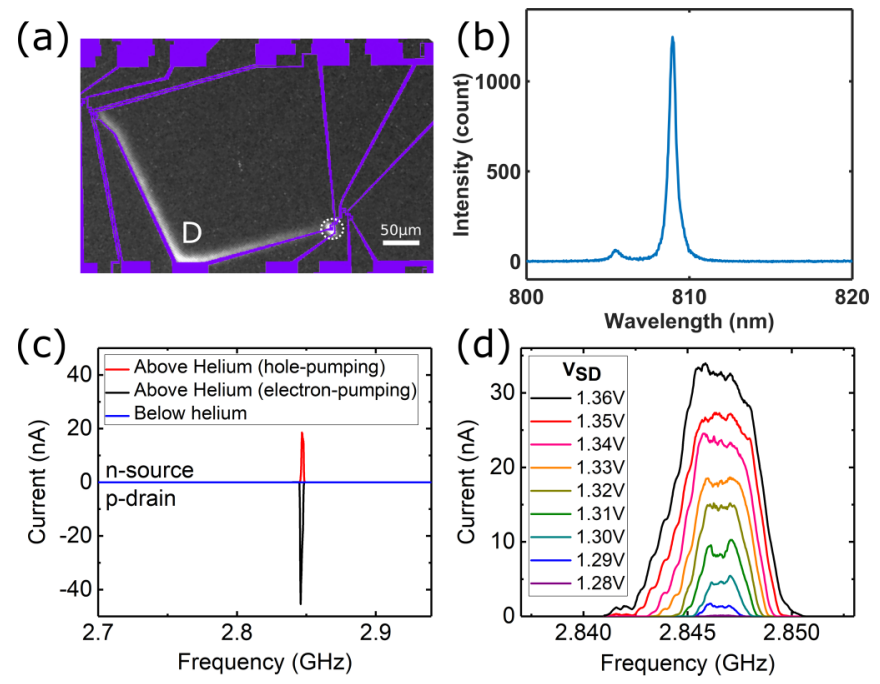

FIG. 4. (a) EL image of device B without SAW at $V_{\mathrm{SiG}}=0 \mathrm{~V}$, $V_{\mathrm{SG} \text { source }}=2 \mathrm{~V}, V_{\mathrm{SG} \text { drain }}=-2 \mathrm{~V}$, and $V_{\mathrm{SD}}=2.5 \mathrm{~V}$, where most of the EL was observed at the $p-n$ junction region, indicated by the dashed circle. The gate design is overlaid (in purple online), aligned from a later optical image. (b) Spectrum taken from the $p-n$ junction enclosed by dashed circle in (a) $\left(V_{\mathrm{SiG}}=0 \mathrm{~V}, V_{\mathrm{SG} \text { source }}=\right.$ $2.5 \mathrm{~V}, V_{\mathrm{SG} \text { drain }}=-2.27 \mathrm{~V}, V_{\mathrm{SD}}=3.1 \mathrm{~V}$ ). (c) SAW-driven current of electrons (holes) across an $n-i-p$ junction from the $n$-type source ( $p$-type drain) vs $f$ (device $\mathrm{A}, V_{\mathrm{SD}}=1.4 \mathrm{~V}$ SAW power $14 \mathrm{dBm}$, $V_{\mathrm{SiG}}=1.08 \mathrm{~V}$ ) when the device is above the liquid-helium surface (no damping). Current disappears on immersion in liquid, which damps the SAW. (d) SAW-driven current for varying S-D bias $V_{\mathrm{SD}}$ [other conditions are as in (c)].

D surface gate [labeled D in Fig. 4(a)] is likely to be due to a lack of confinement for electrons once they reach the region of holes: under such a high S-D bias, electrons with a high kinetic energy can leave the junction region without recombination and eventually recombine with holes under the D surface gate, causing the L-shaped EL (an etched channel alongside this gate prevents the electrons escaping into the central intrinsic region). The measurement was performed at $\sim 20 \mathrm{~K}$. The spectrum obtained at the $p-n$ junction [Fig. 4(b)] has a peak at a wavelength of about $809 \mathrm{~nm}$, as expected for the quantum well in this wafer.

Figure 4(c) shows the SAW-driven current of electrons or holes across the same $n-i-p$ junction using different transducers at either end of the junction. With a positive voltage on the source $\mathrm{BG}\left(V_{\mathrm{BG}}=2.2 \mathrm{~V}\right), V_{\mathrm{SG}}=1.15 \mathrm{~V}$ was applied to the corresponding surface gate in order to induce electrons at the source near the central active region. Similarly, with a negative voltage on the drain $\mathrm{BG}\left(V_{\mathrm{BG}}=-2.0 \mathrm{~V}\right), V_{\mathrm{SG}}=-1.05 \mathrm{~V}$ was applied to the corresponding surface gate in order to induce holes at the drain. A bias of $1.4 \mathrm{~V}$ was applied to the drain relative to the grounded source $\left(V_{\mathrm{SD}}=1.4 \mathrm{~V}\right)$. This is just below the threshold bias [at which the applied bias becomes greater than the band gap $(\sim 1.52 \mathrm{eV})$, causing current flow across the intrinsic region]. The height of the potential barrier at the intrinsic region is then low enough to enable a SAW coming from $\mathrm{S}$ to transfer electrons across the junction from $\mathrm{S}(n)$ to $\mathrm{D}(p)$ or a SAW from the other IDT to carry holes the other way. When pumping electrons, a side-gate voltage 
$V_{\mathrm{SiG}}=1.0 \mathrm{~V}$ was applied relative to $\mathrm{S}(-0.4 \mathrm{~V}$ relative to $\mathrm{D})$ to attract electrons and help extract them from $\mathrm{S}$, so that when a SAW is applied using the IDT at the source $(n)$ end, it can transport electrons to D. Hence, at the resonant frequency of $2.846 \mathrm{GHz}$, there was a large peak in acoustoelectric current across the intrinsic region between $\mathrm{S}$ and $\mathrm{D}$.

Under the same source-drain bias, when the SAW direction was instead from $\mathrm{D}$ to $\mathrm{S}$ and $V_{\mathrm{SiG}}=0.5 \mathrm{~V}[-0.9 \mathrm{~V}$ relative to $\mathrm{D}(p)$ ], holes were pumped from D to S. To confirm that the current was driven by the SAW, the device was immersed in liquid helium and the current was observed to drop to zero, as expected when the SAW is damped by the liquid. Any current driven by the electromagnetic wave itself (crosstalk) would be unaffected. We can rule out the possibility that the current flows because at some stage in the SAW cycle the junction potential is pulled entirely below the Fermi energy by a SAW minimum. This could occur for an $n-i-n$ junction but not for an $n-i-p$ junction, as in the latter case energy is also needed to reach the far side. Also, the SAW half-wavelength was less than the intrinsic region's length. No current quantization was seen like that in Fig. 2 for the $n-i-n$ junction, but it should be possible to quantize SAW transport across the junction if stronger lateral confinement can be obtained to provide a larger charging energy in future experiments [27].

Figure 4(d) shows the current across the $n-i-p$ junction as a function of SAW frequency as the applied bias on the drain is stepped from 1.28 to $1.36 \mathrm{~V}$ in $10 \mathrm{mV}$ steps with a slow sweep rate. The measurement was performed in such a way that the bias was incremented nonmonotonically so that the timedependent drift could be shown to be negligible. A voltage of $V_{\mathrm{SiG}}=1.08 \mathrm{~V}$ was applied to the side gates to assist the SAW to drag electrons from the source to the region of holes at the drain. It is observed that the SAW-driven current increases with source-drain bias. This is expected as the source-drain bias creates an electric field that lowers the potential barrier in the intrinsic region, allowing the SAW to carry more electrons in each minimum. The width of the current peak in frequency also becomes wider as the source-drain bias is increased, unlike that observed for the $n-i-n$ or $p-i-p$ junctions. The resonant peak in the $n-i-p$ case is broadened to a width of $1 \mathrm{MHz}$ (from 2.846 to $2.847 \mathrm{GHz}$ ), still less than the passband of the IDT, as more of the tails of the SAW resonant peak exceeded the pumping threshold amplitude.

We have simulated the electrostatic potential distribution of our $n-i-p$ junction using the same dimensions and voltages as for device A. Figure 5(a) shows conduction and valence bands (dashed lines) at the junction. An external electric field applied by a surface inducing gate pulls the conduction (valence) band below the appropriate Fermi level, inducing free electrons (holes) in the QW. Figure 5(b) illustrates how the slope of the potential barrier in the intrinsic junction region changes with and without a SAW. Applying a nonzero side-gate voltage increases the slope of the potential barrier [Fig. 5(c)], so the required SAW amplitude increases towards $\sim 40 \mathrm{meV}$ for $V_{\mathrm{SiG}} \sim \pm 1 \mathrm{~V}$ (see inset), which is achievable with the current experimental setup. However, because the side-gate voltage required to squeeze one species of carrier attracts the other species, it is necessary to implement a way of reducing unwanted charge carriers in the channel, and hence increase the probability of recombination between
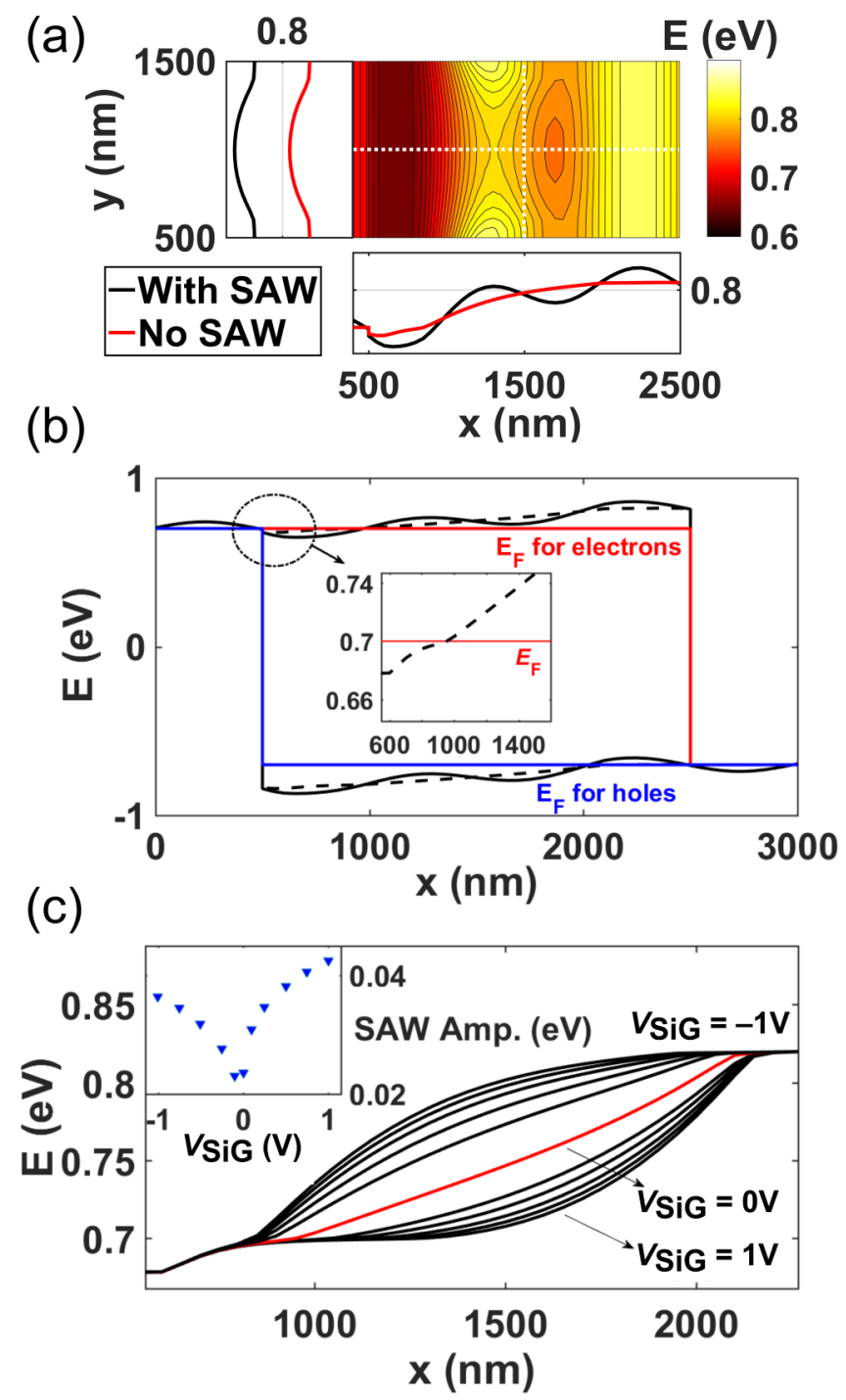

FIG. 5. Modeling an $n-i-p$ junction. (a) 2D electrostatic energy landscape in the $x-y$ plane with added SAW potential $\left(V_{\mathrm{SiG}}=0 \mathrm{~V}\right.$, $V_{\mathrm{SG} \text { source }}=1 \mathrm{~V}, V_{\mathrm{SGdrain}}=-1 \mathrm{~V}$ ). Cross sections in the $x$ and $y$ directions along the white dotted lines are also shown with and without SAW. (b) Energy band structure with added SAW (source bias $V_{\mathrm{S}}=-0.7 \mathrm{~V}$, drain bias $V_{\mathrm{D}}=0.7 \mathrm{~V}$ ). Inset shows enlargement of conduction band (dashed line) when electrons induced below Fermi level. Fermi level is $0.7 \mathrm{~V}$ for electrons (red) and $-0.7 \mathrm{~V}$ for holes (blue). (c) Conduction-band profile through junction with different $V_{\mathrm{SiG}}\left(V_{\mathrm{SG} \text { source }}=1 \mathrm{~V}, V_{\mathrm{SG} \text { drain }}=-1 \mathrm{~V}, V_{\mathrm{S}}=-0.7 \mathrm{~V}, V_{\mathrm{D}}=\right.$ $0.7 \mathrm{~V})$. Inset: Required SAW amplitude vs $V_{\mathrm{SiG}}$.

electrons and holes at the junction. That is why device B and subsequent devices have an additional high-resolution etch on either side of the channel, under the $e$-beam-defined side gates and around the surface gates. Thus, when induced charges are transferred from the source or drain, they should be trapped near the junction before eventually escaping towards the leads, increasing the probability of recombination around the $n-i-p$ junction and consequently allowing photon emission to be more readily detectable there. Etching the regions under the side gates removes any alternative current paths. Such an etched device continued to show acoustoelectric current for several days longer than unetched devices, which lasted 
(a)

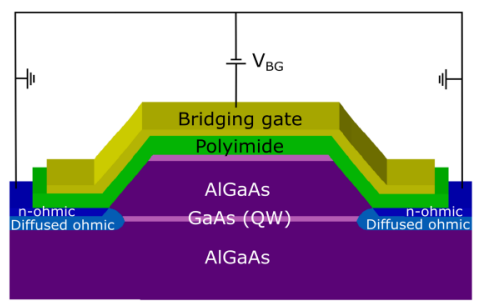

(c)

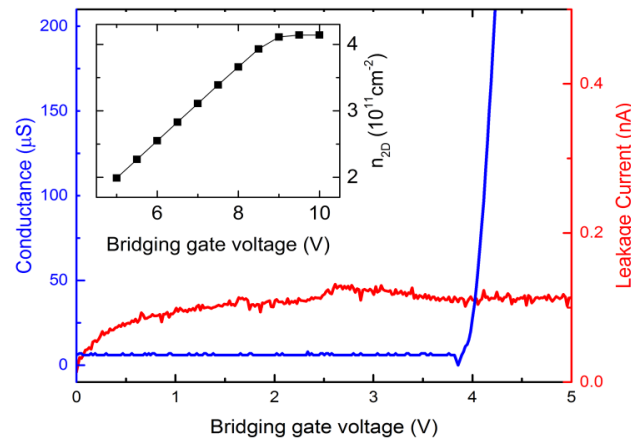

(e) 7.

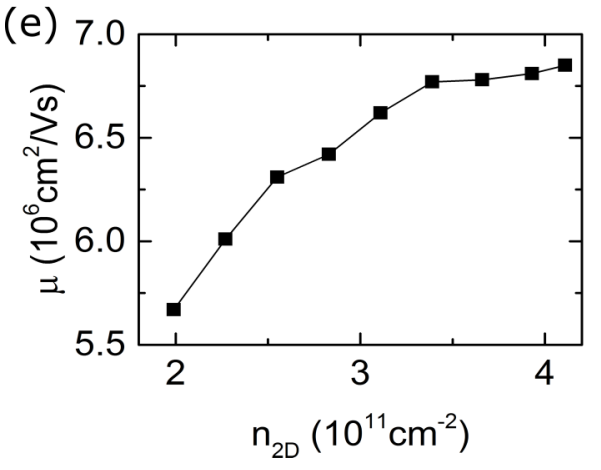

(b)

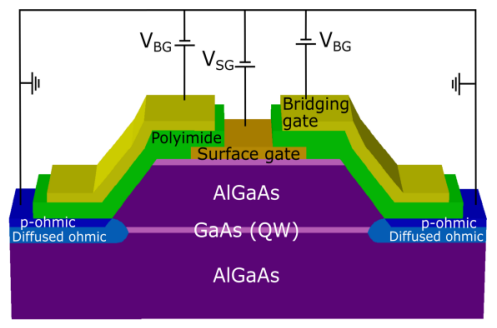

(d)

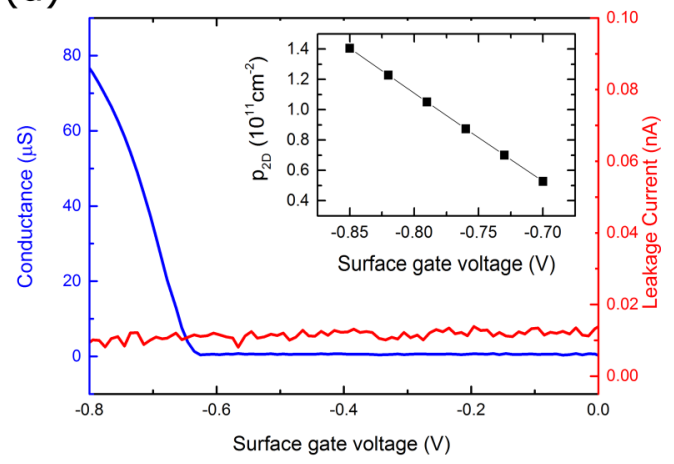

(f)

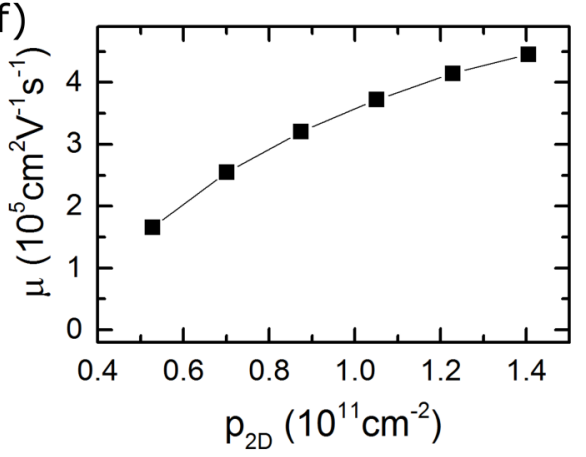

FIG. 6. (a) Schematic diagram of the $n$-type induced device with bridging gate only. The 2DEG is expected to be induced in the quantum well by applying positive bias on the gate. (b) Schematic diagram of the $p$-type induced device with bridging gate and surface gate. The $2 \mathrm{DHG}$ is expected to be induced in the quantum well by applying negative bias on both gates. (c) The measured conductance (blue) and the gate leakage current (red) as a function of the bridging-gate voltage for the $n$-type device at $4 \mathrm{~K}$. Inset: Induced carrier density $n_{2 \mathrm{D}}$ of the device as a function of the bridging-gate voltage from 5 to $10 \mathrm{~V}$ in steps of $0.5 \mathrm{~V}$ at $1.3 \mathrm{~K}$. (d) The measured conductance (blue) and the gate leakage current (red) as a function of the surface-gate voltage for the $p$-type device, when the bridging gate was biased to $-8 \mathrm{~V}$, at $4 \mathrm{~K}$. Inset: Induced carrier density $p_{2 \mathrm{D}}$ of the device as a function of the surface-gate voltage from -0.7 to $-0.85 \mathrm{~V}$ in steps of $-0.03 \mathrm{~V}$ at $1.3 \mathrm{~K}$. (e) Mobility $\mu$ as a function of carrier density for $n$-type induced devices. (f) Mobility as a function of carrier density for $p$-type induced devices.

only a day or so before accumulation of charge in intrinsic regions or at the polyimide insulator interface made current flow impossible [30]. An additional step that can be taken to further increase the lateral confinement in the channel is to add another pair of split side gates in order to allow extra control of the potential along the channel.

\section{CONCLUSIONS}

In summary, using gates to induce high-mobility $2 \mathrm{D}$ electron and hole gases in close proximity, we have fabricated lateral $n-i-n$ and $n-i-p$ junctions, through which a SAW can pump electrons or holes. We observed quantization of this current in an $n-i-n$ junction. Changing the inducing voltages on one of the regions allows SAW-driven recombination of the pumped charges, from which we observe electroluminescence. In principle, this design can also be implemented in other semiconducting 2D systems, such as $\mathrm{MoS}_{2}$, by placing them on a piezoelectric substrate $[31,32]$. This demonstrates the potential development of this device architecture for a versatile, all-solid-state, single-photon source with high repetition rate only limited by emission and collection efficiency and the SAW frequency.

Data related to this paper are available [33].

\section{ACKNOWLEDGEMENTS}

We are grateful for useful discussions with Dr. S. Holmes, Prof. R. Phillips, and Prof. C. Barnes. This work was supported by the European Union Horizon 2020 research and innovation programme under Marie Skłodowska-Curie Grant Agreement No. 642688 (SAWtrain), and the UK EPSRC [Grants No. EP/J003417/1 and No. EP/H017720/1]. A.N. was 

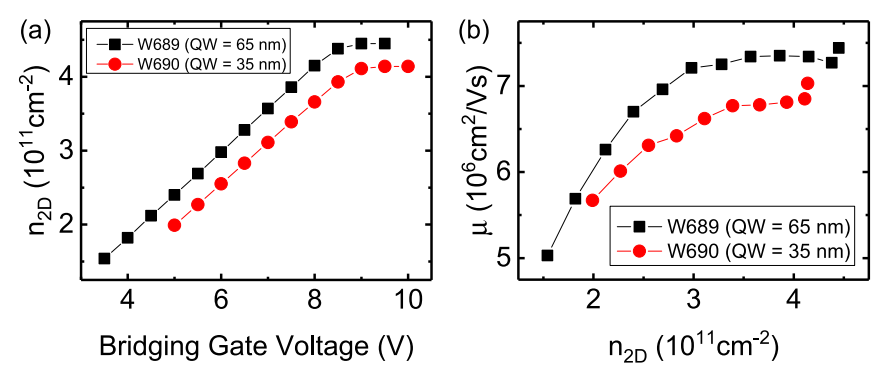

FIG. 7. (a) Induced carrier density $n_{2 \mathrm{D}}$ as a function of the surface-gate voltage and (b) mobility $\mu$ of induced electrons as a function of $n_{2 \mathrm{D}}$ for comparison between devices fabricated from wafers W689 (quantum well width $65 \mathrm{~nm}$ ) and W690 (35 nm). W689 devices have $\mathrm{Al}_{2} \mathrm{O}_{3}$ under the surface gate, while W690 devices are without $\mathrm{Al}_{2} \mathrm{O}_{3}$.

supported by the UK Department for Business, Innovation and Skills. T.-K.H. was supported by the Cambridge Overseas Trust. Y.C. was supported by Newnham College and the Cambridge Overseas Trust.

\section{APPENDIX}

In order to assess the quality and electronic properties of the $2 \mathrm{DEG} / 2 \mathrm{DHG}$ induced in the wafers we used, we fabricated Hall bar devices that induced a $2 \mathrm{DEG} / 2 \mathrm{DHG}$ in the same way as in our actual devices. The carrier density and mobility of the 2DEG/2DHG can only be investigated in this way because the presence of a nonconducting intrinsic region between the source and drain surface gates in the SAW devices precludes the possibility of confirming whether the surface gates are operating simply by measuring the current flow between them (since no current should be able to flow through the intrinsic region). By applying a positive bias on the bridging gate of about $4 \mathrm{~V}$, a $2 \mathrm{DEG}$ was formed on the device with negligible leakage current of $100 \mathrm{pA}$ at $4 \mathrm{~K}$ [see Fig. 6(c)]. The carrier density can be determined from the slope of the Hall resistance at low perpendicular magnetic field $B$. The mobility is then determined from the carrier density and the four-terminal longitudinal resistance at $B=$ 0 . As shown in the inset of Fig. 6(c), the induced electron density $n_{2 \mathrm{D}}$ varies linearly with the bridging gate bias up to $4.1 \times 10^{11} \mathrm{~cm}^{-2}$ before it saturates due to the accumulation of electrons at the GaAs surface underneath the polyimide.

The depth of the 2DEG from the surface $(\sim 110 \mathrm{~nm})$ is estimated from the capacitance where the gradient $0.558 \times$ $10^{11} \mathrm{~cm}^{-2} \mathrm{~V}^{-1}$ is taken from this plot and the thickness of the polyimide is measured to be $298 \pm 7 \mathrm{~nm}$. This approximation suggests that for a given applied bias the induced 2DEG is located near the GaAs/AlGaAs heterointerface within the quantum well (110-145 nm below the surface) with electron density $4.1 \times 10^{11} \mathrm{~cm}^{-2}$ and mobility $6.9 \times 10^{6} \mathrm{~cm}^{2} \mathrm{~V}^{-1} \mathrm{~s}^{-1}$ at $1.3 \mathrm{~K}$. In order for recombination to occur between the induced electrons and holes in our $n-i-p$ junction devices, it is obviously necessary for both to be induced in the same quantum well.

Figure 6(d) indicates the threshold surface-gate bias, about $-0.63 \mathrm{~V}$ for inducing a $2 \mathrm{DHG}$ in the Hall bar. There was negligible leakage current $(50 \mathrm{pA})$ at $4 \mathrm{~K}$, where the bridging gate was biased to $-8 \mathrm{~V}$ and the threshold bridging-gate bias
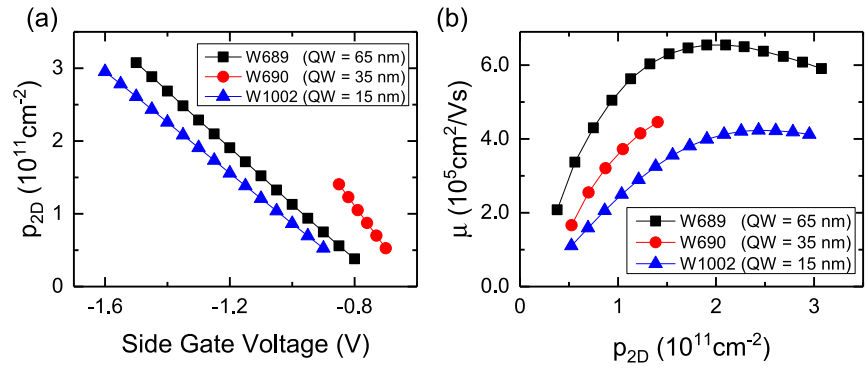

FIG. 8. (a) Induced carrier density $p_{2 \mathrm{D}}$ as a function of the surface gate voltage and (b) mobility $\mu$ of induced holes as a function of $p_{2 \mathrm{D}}$ for comparison between devices fabricated from wafers W689 (quantum well width $=65 \mathrm{~nm}), \mathrm{W} 690(35 \mathrm{~nm})$, and W1002 $(15 \mathrm{~nm})$. W689 and W1002 devices have $\mathrm{Al}_{2} \mathrm{O}_{3}$ under the surface gate, while W690 is without $\mathrm{Al}_{2} \mathrm{O}_{3}$.

for inducing a $2 \mathrm{DHG}$ was $-6 \mathrm{~V}$ in this case. In order to test the surface gates, it is necessary to measure between two ohmic contacts that are located in different regions where the bridging gates are isolated from each other and can be independently addressed [see Fig. 6(b)]. In this way, charge carriers can only flow between the bridging gates when a negative voltage is also applied to the surface gates, which drags the holes under the surface gate (in this case a Hall bar). The charge density at the ohmic contacts can be independently tuned from that under the surface gate, because the surface gate screens the electric field from the bridging gate. The inset of Fig. 6(d) shows that the measured hole carrier density has a direct linear dependence on the applied surface-gate bias with gradient $5.86 \times 10^{11} \mathrm{~cm}^{-2} \mathrm{~V}^{-1}$. Four-terminal Hall-bar measurements at $1.3 \mathrm{~K}$ show that the $2 \mathrm{DHG}$ was successfully induced at a depth of around $114 \mathrm{~nm}$, which is in the expected quantum well, and give a mobility of $4.5 \times 10^{5} \mathrm{~cm}^{2} \mathrm{~V}^{-1} \mathrm{~s}^{-1}$ at a hole carrier density of $1.48 \times 10^{11} \mathrm{~cm}^{-2}$. Compared to the bridging-gate system, since the surface gate sits directly on the device surface, the measurement of the depth of the $2 \mathrm{DHG}$ from the surface is more precise, due to the absence of a nonuniform thickness of insulator. Moreover, when bias is applied, any possible slow accumulation of charge at the surface is avoided as it discharges to the gate (so that the stable lifetime for measurements of the device is lengthened).

The mobility at different densities was further investigated for both $n$-type and $p$-type induced wafers using wafers with different quantum-well widths. Figures 7 and 8 show the carrier densities and mobilities for each device from different wafers measured at a temperature of $1.3 \mathrm{~K}$. The $n$-type devices in Fig. 7 have polyimide thicknesses of $285 \pm 7 \mathrm{~nm}$ for W689 (65 nm QW) and $298 \pm 7 \mathrm{~nm}$ for W690 (35 nm QW).

For the $p$-type devices in Fig. 8, the voltage applied to the bridging gates was $-5,-8$, and $-8 \mathrm{~V}$ for devices on wafers W689 (65 nm QW), W690 (35 nm QW), and W1002 (15 nm QW), respectively. Since W689 and W1002 have $\mathrm{Al}_{2} \mathrm{O}_{3}$ under the surface gate, while W690 was fabricated without $\mathrm{Al}_{2} \mathrm{O}_{3}$, a comparison can be made between devices with or without $\mathrm{Al}_{2} \mathrm{O}_{3}$. From the plot of induced carrier density as a function of surface-gate voltage, the gradient $S_{\text {data }}$ for the devices on W689 and W1002 was found to be $-3.86 \times 10^{11}$ and $-3.48 \times 10^{11} \mathrm{~cm}^{-2} \mathrm{~V}^{-1}$, respectively. These are smaller compared to the gradient measured for the 


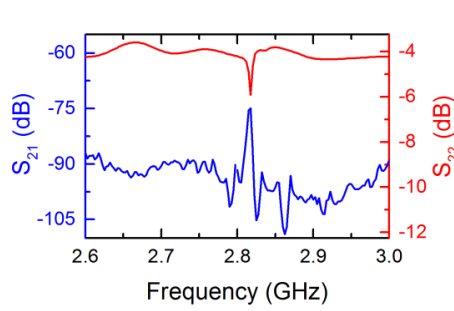

(a)

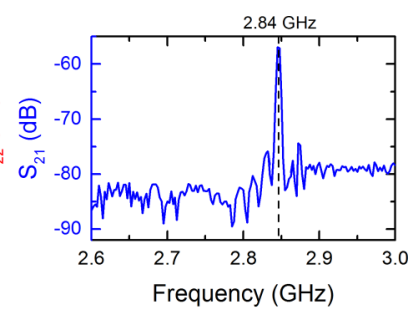

(b)
FIG. 9. Transducer measurements for the device (a) at room temperature, and (b) at $4 \mathrm{~K} . S_{21}$ (insertion loss) and $S_{22}$ (return loss) as a function of frequency are plotted in blue and red, respectively.

device from $\mathrm{W} 690$ due to the presence of $\mathrm{Al}_{2} \mathrm{O}_{3}$. On the other hand, mobility is not affected by the presence of $\mathrm{Al}_{2} \mathrm{O}_{3}$, and instead reveals the effect of different quantum-well widths on mobility. It was found that mobility is lower for narrower quantum wells. However, it is noted that W1002 was grown at a different time so the MBE chamber quality could also have been different.

The bridging and surface gates are operated by applying a DC bias using IOTech DACs and Keithley 2400 source meters. The currents through the induced 2DEG and 2DHG were measured simultaneously via two Stanford SR570 lownoise current preamplifiers each connected at opposite ohmic contacts of the device. By employing one preamplifier each for the source and drain, any leakage current shows up as a difference between the current measured between the two preamplifiers, allowing for the fact that they should have opposite signs.

At the resonant frequency of $2.848 \mathrm{GHz}$, the acoustoelectric current across the isolated source and drain surface gates was found to be approximately $3.5 \mathrm{nA}$ with an applied SAW $\mathrm{RF}$ power of $14 \mathrm{dBm}$ at $4 \mathrm{~K}$. The resonant frequency is in good agreement with that found from $\mathrm{S}_{21}$ measurements, and is expected to be close to $2.8 \mathrm{GHz}$ given a transducer finger periodicity of $1 \mu \mathrm{m}$ and the speed of sound in GaAs of $\sim 2800 \mathrm{~ms}^{-1}$, so it is likely that the current is due to SAW-driven transport of electrons across the $n-i$ - $n$ junction.

Before measuring the device electrically at low temperatures $(4 \mathrm{~K}$ or $300 \mathrm{mK}$ ), the SAW transducers in the device were assessed using an RF network analyzer at room temperature (see Fig. 9), which allows the measurement of four scattering parameters $S_{11}, S_{12}, S_{21}$, and $S_{22}$. $S_{12}$ or $S_{21}$ is also known as the insertion loss, which represents the loss in power transmitted from one SAW transducer to that on the other side of the chip (or vice versa), and is described by $S_{\mathrm{ti}}=10 \log _{10} \frac{P_{\mathrm{i}}}{P_{\mathrm{t}}}$, where $P_{\mathrm{i}}$ is the incident power, and $P_{\mathrm{t}}$ is the transmitted power after the device is inserted. Similarly, the return loss $\left(S_{11}\right.$ and $S_{22}$, representing the reflection from each transducer) is given by $S_{\mathrm{ii}}=10 \log _{10} \frac{P_{\mathrm{i}}}{P_{\mathrm{r}}}$, where $P_{\mathrm{r}}$ is the power reflected from the device. Theoretically, the resonant

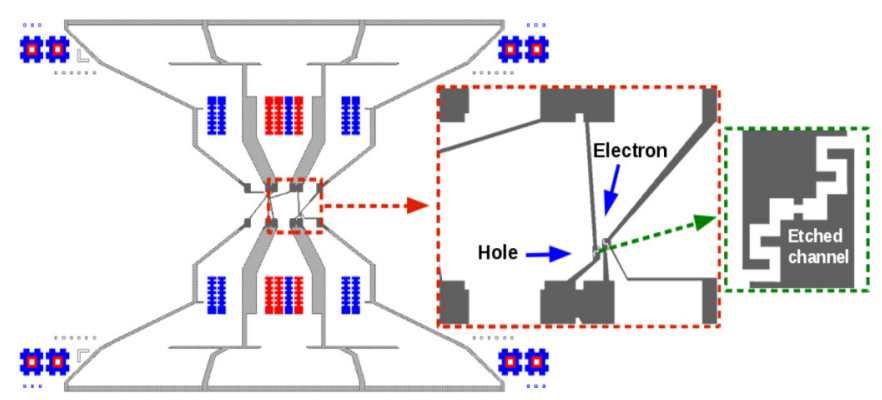

FIG. 10. Schematic of the etched channel surrounding the $n-i-p$ junction for device B, as shown in Fig. 4(a) in the main text.

frequency of the transducers can be calculated from the designed periodicity of the interdigitated fingers in the transducers following the equation $f_{\text {SAW }} \lambda_{\text {IDT }}=v_{\text {SAW }}$, where $v_{\text {SAW }}$ is the SAW velocity in the material. In our device, the finger periodicity of $1 \mu \mathrm{m}$ and SAW velocity of $2800 \mathrm{~ms}^{-1}$ give a resonant frequency of $2.80 \mathrm{GHz}$. The resonant frequency has to be determined more precisely by measuring the insertion (return) loss, which exhibits a peak (dip) at the resonant frequency because the reflection is least when a SAW takes power to the other transducer, giving a peak in transmission.

For our devices, a $10 \mathrm{dBm}$ applied RF signal gives an insertion loss $\left(S_{21}=S_{12}\right)$ of $-75 \mathrm{~dB}$ at the resonant frequency of $2.82 \mathrm{GHz}$, close to the calculated resonant frequency, with a background signal of about $-90 \mathrm{~dB}$. The background signal away from the resonance is most likely due to electromagnetic crosstalk, which is minimized by shielding the device using a lid on the sample holder. The return loss $\left(S_{11}=S_{22}\right)$ shows a dip of $-5.9 \mathrm{~dB}$ at $2.82 \mathrm{GHz}$ (with a background signal of about $-4 \mathrm{~dB}$ ), in good agreement with the resonant frequency measured from the insertion loss. The increase in amplitude of the $S_{21}$ SAW peak from $-75 \mathrm{~dB}$ at room temperature to $-57 \mathrm{~dB}$ at $4 \mathrm{~K}$ is likely to be due to the freezing out of surface carriers at low temperatures. The resonant frequency is also shifted to a higher frequency of $2.84 \mathrm{GHz}$, due to the contraction of the transducers at low temperatures, which reduces the transducer period and hence the SAW wavelength.

A single-finger design was chosen for the transducers as this provides a narrow peak with $Q \approx 500$. $Q$ is calculated from $Q=f /$ FWHM, where $f$ is the resonant frequency and FWHM is the full-width at half-maximum of the peak. This $Q$ is suitable for continuous or long pulsed ( $>1 \mu \mathrm{s}$ ) SAW measurements that lead to increased quality of the quantized acoustoelectric current [34].

Figure 10 shows a schematic of device B mentioned in the main text, where the details of the etched channel is shown. This etched channel helps confine the carriers to the $n-i-p$ junction in order to promote electron-hole recombination at this junction.
[1] A. J. Shields, Nat. Photonics 1, 215 (2007).

[2] C. L. Foden, V. I. Talyanskii, G. J. Milburn, M. L. Leadbeater, and M. Pepper, Phys. Rev. A 62, 011803(R) (2000).

[3] M. M. de Lima, R. Hey, J. A. H. Stotz, and P. V. Santos, Appl. Phys. Lett. 84, 2569 (2004).
[4] C. H. W. Barnes, J. M. Shilton, and A. M. Robinson, Phys. Rev. B 62, 8410 (2000).

[5] R. P. G. McNeil, M. Kataoka, C. J. B. Ford, C. H. W. Barnes, D. Anderson, G. A. C. Jones, I. Farrer, and D. A. Ritchie, Nature (London) 477, 439 (2011). 
[6] S. Hermelin, S. Takada, M. Yamamoto, S. Tarucha, A. D. Wieck, L. Saminadayar, C. Bauerle, and T. Meunier, Nature (London) 477, 435 (2011).

[7] T. Aref, P. Delsing, M. K. Ekström, A. F. Kockum, M. V. Gustafsson, G. Johansson, P. Leek, E. Magnusson, and R. Manenti, in Superconducting Devices in Quantum Optics, edited by R. Hadfield, G. Johansson, Quantum Science and Technology (Springer, Cham, 2016).

[8] M. J. A. Schuetz, E. M. Kessler, G. Giedke, L. M. K. Vandersypen, M. D. Lukin, and J. L. Cirac, Phys. Rev. X 5, 031031 (2015).

[9] B. Bertrand, S. Hermelin, S. Takada, M. Yamamoto, S. Tarucha, A. Ludwig, A. Wieck, C. Bäuerle, and T. Meunier, Nat. Nanotechnol. 11, 672 (2016).

[10] B. Bertrand, S. Hermelin, P.-A. Mortemousque, S. Takada, M. Yamamoto, S. Tarucha, A. Ludwig, A. D. Wieck, C. Bäuerle, and T. Meunier, Nanotechnology 27, 214001 (2016).

[11] S. Takada, H. Edlbauer, H. V. Lepage, J. Wang, P.-A. Mortemousque, G. Georgiou, C. H. W. Barnes, C. J. B. Ford, M. Yuan, P. V. Santos, X. Waintal, A. Ludwig, A. D. Wieck, M. Urdampilleta, T. Meunier, and C. Bäuerle, Nat. Commun. 10, 4557 (2019).

[12] H. Kosaka, T. Inagaki, Y. Rikitake, H. Imamura, Y. Mitsumori, and K. Edamatsu, Nature (London) 457, 702 (2009).

[13] J. M. Shilton, V. I. Talyanskii, M. Pepper, D. A. Ritchie, J. E. F. Frost, C. J. B. Ford, C. G. Smith, and G. A. C. Jones, J. Phys.: Condens. Matter 8, L531 (1996).

[14] V. I. Talyanskii, J. M. Shilton, M. Pepper, C. G. Smith, C. J. B. Ford, E. H. Linfield, D. A. Ritchie, and G. A. C. Jones, Phys. Rev. B 56, 15180 (1997).

[15] J. Kim, O. Benson, H. Kan, and Y. Yamamoto, Nature (London) 397, 500 (1999).

[16] Z. Yuan, B. Kardynal, R. M. Stevenson, A. J. Shields, C. J. Lobo, K. Cooper, N. S. Beattie, D. A. Ritchie, and M. Pepper, Science 295, 102 (2002).

[17] T. Hosey, V. Talyanskii, S. Vijendran, G. A. C. Jones, M. B. Ward, D. C. Unitt, C. E. Norman, and A. J. Shields, Appl. Phys. Lett. 85, 491 (2004).

[18] M. Cecchini, V. Piazza, F. Beltram, D. G. Gevaux, M. B. Ward, A. J. Shields, H. E. Beere, and D. A. Ritchie, Appl. Phys. Lett. 86, 241107 (2005).
[19] S. J. Smith, G. R. Nash, M. F. Lewis, C. J. Bartlett, L. Buckle, M. T. Emeny, and T. Ashley, Appl. Phys. Lett. 89, 111118 (2006).

[20] B. Kaestner, J. Wunderlich, J. Sinova, and T. Jungwirth, Appl. Phys. Lett. 88, 091106 (2006).

[21] J. R. Gell, M. B. Ward, A. J. Shields, P. Atkinson, S. P. Bremner, D. Anderson, M. Kataoka, C. H. W. Barnes, G. A. C. Jones, and D. A. Ritchie, Appl. Phys. Lett. 91, 013506 (2007).

[22] V. T. Dai, S. D. Lin, S. W. Lin, Y. S. Lee, Y. J. Zhang, L. C. Li, and C. P. Lee, Opt. Express 22, 3811 (2014).

[23] G. De Simoni, V. Piazza, L. Sorba, G. Biasiol, and F. Beltram, Appl. Phys. Lett. 94, 121103 (2009).

[24] C. J. B. Ford, Phys. Status Solidi B 254, 1600658 (2017).

[25] H. Hou, Y. Chung, G. Rughoobur, T.-K. Hsiao, A. Nasir, A. Flewitt, J. P. Griffiths, I. Farrer, D. A. Ritchie, and C. J. B. Ford, J. Phys. D: Appl. Phys. 51, 244004 (2018).

[26] R. J. Schneble, M. Kataoka, C. J. B. Ford, C. H. W. Barnes, D. Anderson, G. A. C. Jones, I. Farrer, D. A. Ritchie, and M. Pepper, Appl. Phys. Lett. 89, 122104 (2006).

[27] M. Kataoka, C. H. W. Barnes, H. E. Beere, D. A. Ritchie, and M. Pepper, Phys. Rev. B 74, 085302 (2006).

[28] M. R. Astley, M. Kataoka, C. J. B. Ford, C. H. W. Barnes, D. Anderson, G. A. C. Jones, I. Farrer, D. A. Ritchie, and M. Pepper, Phys. Rev. Lett. 99, 156802 (2007).

[29] M. Büttiker, Phys. Rev. B 41, 7906 (1990).

[30] T.-K. Hsiao, A. Rubino, Y. Chung, S.-K. Son, H. Hou, J. Pedrós, A. Nasir, G. Éthier-Majcher, M. Stanley, R. T. Phillips, T. A. Mitchell, J. P. Griffiths, I. Farrer, D. A. Ritchie, and C. J. B. Ford, arXiv:1901.03464.

[31] A. Hernández-Mínguez, Y.-T. Liou, and P. V. Santos, J. Phys. D: Appl. Phys. 51, 383001 (2018).

[32] A. R. Rezk, B. Carey, A. F. Chrimes, D. W. M. Lau, B. C. Gibson, C. Zheng, M. S. Fuhrer, L. Y. Yeo, and K. Kalantarzadeh, Nano Lett. 16, 849 (2016).

[33] https://doi.org/10.17863/CAM.45925.

[34] M. Kataoka, C. H. W. Barnes, D. Anderson, G. A. C. Jones, H. E. Beere, D. A. Ritchie, and M. Pepper, J. Appl. Phys. 100, 063710 (2006). 